\title{
Terahertz Stimulated Emission from Silicon Doped by Hydrogenlike Acceptors
}

\author{
S. G. Pavlov, ${ }^{1}$ N. Deßmann, ${ }^{2}$ V. N. Shastin, ${ }^{3,4}$ R. Kh. Zhukavin, ${ }^{3}$ B. Redlich, ${ }^{5,6}$ A. F. G. van der Meer, ${ }^{5,6}$ \\ M. Mittendorff, ${ }^{7}$ S. Winnerl, ${ }^{7}$ N. V. Abrosimov, ${ }^{8}$ H. Riemann, ${ }^{8}$ and H.-W. Hübers ${ }^{1,2}$ \\ ${ }^{1}$ Institute of Planetary Research, German Aerospace Center (DLR), 12489 Berlin, Germany \\ ${ }^{2}$ Institut für Optik und Atomare Physik, Technische Universität Berlin, 10623 Berlin, Germany \\ ${ }^{3}$ Institute for Physics of Microstructures, Russian Academy of Sciences, 603950 Nizhny Novgorod, Russia \\ ${ }^{4}$ Nizhny Novgorod State University, 603950 Nizhny Novgorod, Russia \\ ${ }^{5}$ FOM Institute for Plasma Physics Rijnhuizen, 3430 BE Nieuwegein, The Netherlands \\ ${ }^{6}$ Institute for Molecules and Materials, Radboud University Nijmegen, \\ 6525 ED Nijmegen, The Netherlands \\ ${ }^{7}$ Helmholtz-Zentrum Dresden-Rossendorf, 01328 Dresden, Germany \\ ${ }^{8}$ Leibniz Institute for Crystal Growth, 12489 Berlin, Germany
}

(Received 27 September 2013; revised manuscript received 10 February 2014; published 10 April 2014)

\begin{abstract}
Stimulated emission in the terahertz frequency range has been realized from boron acceptor centers in silicon. Population inversion is achieved at resonant optical excitation on the $1 \Gamma_{8}^{+} \rightarrow 1 \Gamma_{7}^{-}, 1 \Gamma_{6}^{-}, 4 \Gamma_{8}^{-}$ intracenter transitions with a midinfrared free-electron laser. Lasing occurs on two intracenter transitions around $1.75 \mathrm{THz}$. The upper laser levels are the $1 \Gamma_{7}^{-}, 1 \Gamma_{6}^{-}$, and $4 \Gamma_{8}^{-}$states, and the lower laser level for both emission lines is the $2 \Gamma_{8}^{+}$state. In contrast to $n$-type intracenter silicon lasers, boron-doped silicon lasers do not involve the excited states with the longest lifetimes. Instead, the absorption cross section for the pump radiation is the dominating factor. The four-level lasing scheme implies that the deepest even-parity boron state is the $2 \Gamma_{8}^{+}$state and not the $1 \Gamma_{7}^{+}$split-off ground state, as indicated by other experiments. This is confirmed by infrared absorption spectroscopy of Si:B.
\end{abstract}

DOI: 10.1103/PhysRevX.4.021009

\section{INTRODUCTION}

Transitions between energy levels of an impurity in a host crystal are the basis of many solid-state lasers [1]. Examples range from the ruby laser, the first-ever-made laser, to the very powerful Nd:YAG laser. The very long lifetimes of particular impurity levels, which can be as long as a few milliseconds, enable efficient laser schemes where population inversion is obtained between a long-living upper level and a lower level with a much shorter lifetime. The concept of using optical transitions between excited states of impurity atoms in semiconductors with indirect band gap, such as silicon, has been explored in recent years. These approaches are based on substitutional hydrogenlike impurity centers in silicon [2,3] and rare-earth atoms embedded in a silicon crystal $[4,5]$. Although the impurity states, which are involved in dipole-allowed optical transitions in silicon, have typical lifetimes on the picosecond to nanosecond scale, population inversion can be achieved due to large optical cross sections that enable efficient optical pumping. This compensates for the short lifetime of the upper laser level. Together with near-infrared Raman-type

Published by the American Physical Society under the terms of the Creative Commons Attribution 3.0 License. Further distribution of this work must maintain attribution to the author(s) and the published article's title, journal citation, and DOI.
Subject Areas: Optics, Semiconductor Physics

lasers [6,7] and hybrid III-V silicon evanescent lasers [8], these are the only lasers that are based on silicon.

Stimulated terahertz $(\mathrm{THz})$ emission from dipoleallowed intracenter transitions as well as Raman lasing has been demonstrated for all group- $\mathrm{V}$ substitutional donors in silicon [9]. These lasers operate at low temperatures $(<30 \mathrm{~K})$ either with optical excitation directly into one of the excited states or by photoexcitation into the conduction band. All laser schemes in $n$-Si are based on the specific electron-phonon interaction, which leads to different relaxation rates for the excited impurity states. Evenparity states always relax much faster into the even-parity ground state than into odd-parity states [10]. Therefore, at nonequilibrium conditions, odd-parity states are more populated than the adjacent lower-lying even-parity states and laser emission occurs from dipole-allowed odd $\rightarrow$ even intracenter optical transitions. Unless particular donor states are in resonance with intervalley phonons [11], the upper level of the intracenter laser is always the odd-parity state with the longest lifetime, which is the state with the largest energy gap to its neighboring even-parity state. As a result, only four-level laser schemes can be realized in $n$-Si and the laser transition is between the lowest odd-parity and the lowest even-parity state [11]. Single-phonon electronic interaction with a zone-centered optical phonon (phonon energy $h \omega_{\text {LTO }} \sim 64 \mathrm{meV}$ ) is weak in silicon and does not contribute to the nonradiative relaxation of bound and free 
electrons. This allows use of radiation sources with photon energies exceeding $h \omega_{\mathrm{LTO}}$, such as a $\mathrm{CO}_{2}$ laser, to populate excited states of hydrogenlike impurities [2]. Resonant pumping in excited impurity states (photon energy less than $\left.h \omega_{\text {LTO }}\right)$ results in even more efficient lasing schemes [12]. While $n$-Si lasers have been realized with a number of dopants [9], a $p$-Si laser has not yet been demonstrated. Only spontaneous emission under $\mathrm{CO}_{2}$-laser pumping as well as electroluminescence has been observed from electrically excited Si:B [13].

The energy structure of hydrogenlike acceptors in silicon has some peculiarities that are related to the strong spinorbit (SO) coupling of the valence subbands (labeled $\Gamma_{8}^{+}$ and $\Gamma_{7}^{+}[14,15]$, Fig. 1). The SO coupling breaks the parabolicity of the $\Gamma_{7}^{+}$subband and the spectrum of acceptor states related to the $\Gamma_{7}^{+}$valence band (VB) is modified. But it remains resonant to the continuum of the states in the $\Gamma_{8}^{+}$VB. Theoretical calculations suggest a strong admixing of hydrogenlike wave functions from the lower $1 \Gamma_{8}^{+} \mathrm{VB}$ to the resonant states bound to the bottom of the upper $1 \Gamma_{7}^{+} \mathrm{VB}$. This induces large shifts of the resonant state energies and it makes the entire impurity spectrum nonhydrogenlike $[15,16]$. For $\mathrm{Si}: \mathrm{B}$, for instance, the calculated shift is in the order of the spin-orbit energy of $\Delta_{\text {SO }} \approx$ $44 \mathrm{meV}$ that would result in the $1 \Gamma_{7}^{+}$state being localized in the silicon band gap with a binding energy of $E_{\Gamma 7+} \approx$ $-22 \mathrm{meV}[15,16]$. This is larger than the binding energy of any other excited boron state (Fig. 1).

However, a controversy exists about the ground state of boron in the $\Gamma_{7}^{+}$subband, which, according to some experiments [17-19], is located in the energy gap of silicon at an energy of $E_{S} \approx-23 \mathrm{meV}$, while other experiments $[13,20-22]$ do not show any evidence for this. Since optical transitions between the subband's ground states are forbidden in the dipole approximation, it is not possible to directly measure the $1 \Gamma_{7}^{+}$state energy $\mathrm{E}_{1 \Gamma 7+}$ by lowtemperature absorption spectroscopy. Wright and Mooradian [17] and Cherlow et al. [18] attributed a feature at $E_{S} \approx 23 \mathrm{meV}$ in the Raman spectrum of a Si:B sample (doping concentration $N_{B} \sim 2 \times 10^{16} / \mathrm{cm}^{3}$ ) to the resonant $1 \Gamma_{7}^{+} \rightarrow 1 \Gamma_{8}^{+}$transition resulting in $E_{1 \Gamma 7+} \approx 23 \mathrm{meV}$, which is in the band gap (lower dashed line in Fig. 1). Chandrasekhar et al. assigned the only spectral feature at $22.77 \mathrm{meV}$ in the absorption spectrum of a heavily doped $\left(N_{B} \sim 10^{17} / \mathrm{cm}^{3}\right)$ Si:B sample as a candidate for a transition ending in the localized $1 \Gamma_{7}^{+}$state [19]. Thewalt, in contrast, did not observe any line with an energy around $23 \mathrm{meV}$ in photoluminescence spectra obtained from floatzone-refined Si:B $\left(N_{B} \sim 2.2 \times 10^{15} \mathrm{~cm}^{-3}\right)$. He could not confirm the existence of the $1 \Gamma_{7}^{+}$state in the band gap [20]. However, he observed a full set of transitions to other evenparity states. As an alternative explanation, Jones et al. attributed the $22.81 \mathrm{meV}$ line in the absorption spectra of Si:B to a carbon-boron pair (B-X) center with a binding energy of $-37.1 \mathrm{meV}$ [21]. Also, electroluminescence
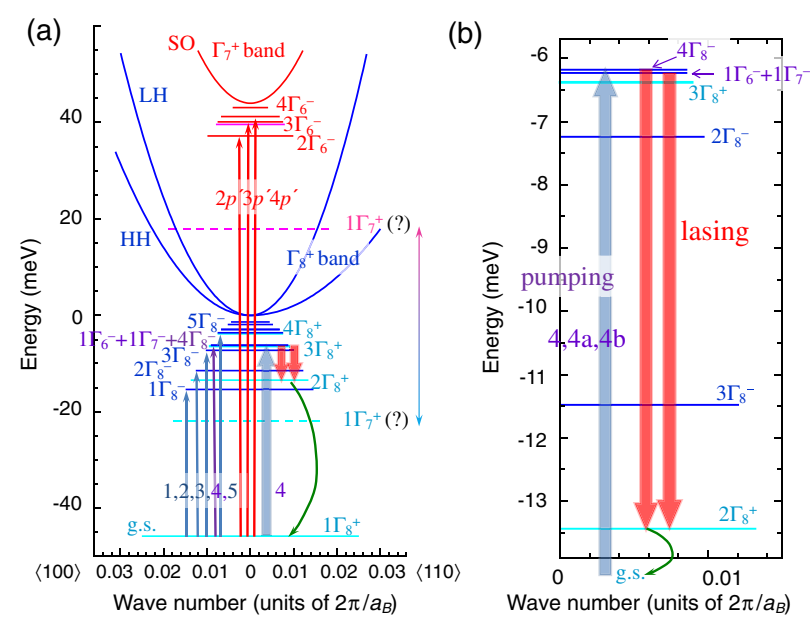

FIG. 1. (a) Band diagram of boron impurity levels in silicon (HH, heavy hole subband; LH, light hole subband; SO, spin-orbit split-off subband). Negative energies correspond to the band gap. The narrow arrows up show dipole-allowed boron absorption transitions (ground state $\rightarrow \Gamma_{8}^{+}$subband: lines 1, 2, 3, 4, 5) (ground state $\rightarrow \Gamma_{7}^{+}$subband: lines $2^{\prime}, 3^{\prime}, 4^{\prime}$ ), which are regularly observed in low-temperature absorption spectroscopy [14]. The $1 \Gamma_{7}^{+}$ground state of the split-off state series $\left(\Gamma_{7}^{+}\right.$band) is expected to occur in between the two horizontal dashed lines: between resonant to the $\Gamma_{8}^{+}$band location [14] and the theoretically predicted in the band gap [15]. The light blue dashed line is the position where the $1 \Gamma_{7}^{+}$ground state should appear according to Raman spectroscopy $[17,18]$. (b) Si:B laser scheme. The bold straight arrows indicate the transitions for stimulated emission (red downward arrows) under optical pumping (bold blue upward arrow). Note that the laser transitions end in the lowest even-parity state. The curved green arrow indicates nonradiative relaxation.

spectra observed for boron acceptors [13] and phosphorus donors [22] indicate that the $1 \Gamma_{7}^{+}$state is not in the band gap. Luminescence lines of Si:P end in the same split-off ground state, which is part of the four-level laser scheme in optically pumped Si:P [2]. In contrast, all electroluminescence lines observed from $\mathrm{Si}: \mathrm{B}$ [13] end in the boron ground state $1 \Gamma_{8}^{+}$. This indicates that in Si:B population inversion might be achieved in a three-level scheme, not requiring a $1 \Gamma_{7}^{+}$state in the band gap.

The controversy about the $1 \Gamma_{7}^{+}$state has some implications for potential laser mechanisms in Si:B. Assuming that the $1 \Gamma_{7}^{+}$state is localized in the silicon band gap, a fourlevel laser scheme with the $1 \Gamma_{8}^{-} \rightarrow 1 \Gamma_{7}^{+}$laser transition can be expected in optically pumped $\mathrm{Si}$ :B. In this case, optical pumping is possible with photons of energy equal to or larger than $E_{1 \Gamma 8_{+}}-E_{1 \Gamma 8_{-}} \approx 30.4 \mathrm{meV}$ and the most effective resonant pumping should occur for the $1 \Gamma_{8}^{+} \rightarrow 1 \Gamma_{8}^{-}$ transition at $30.4 \mathrm{meV}$, which has a large oscillator strength $\left(1.77 \times 10^{-2}[16]\right)$ and the longest lifetime of all excited states [23]. If the $1 \Gamma_{7}^{+}$state is not in the band gap, this laser mechanism is not possible. 
Here, we report on the realization of population inversion and lasing in $p$-Si, namely silicon doped with boron acceptors. Lasing is achieved at a lattice temperature of approximately $5 \mathrm{~K}$ by resonant infrared optical pumping into excited boron states. The large oscillator strengths of the closely spaced boron absorption lines enable gain at transitions between particular excited states. The laser scheme is completely different from those known for $n$-type $\mathrm{THz}$ intracenter silicon lasers [9] and it does not involve emission lines observed in electrically excited Si:B [13]. This indicates that the $1 \Gamma_{7}^{+}$state is not in the band gap.

\section{EXPERIMENTAL PROCEDURE}

Several Si:B crystals were grown by float zone as well as Czochralski techniques in $\langle 100\rangle$ or $\langle 111\rangle$ directions. The doping concentration is in the range of $N_{B} \sim(1-5) \times$ $10^{15} \mathrm{~cm}^{-3}$. This concentration was chosen because it covers the optimal doping range of $n$-Si lasers [9]. The crystals are characterized by measuring their absorption spectra with a Fourier transform infrared (FTIR) spectrometer.

The Si samples for obtaining stimulated emission have dimensions of approximately $7 \times 7 \times 5 \mathrm{~mm}^{3}$. The surfaces are optically polished to form a high-Q resonator operating on total internal reflection modes. The samples are mounted in a cryogenic dipstick that is immersed in a liquid helium transport vessel. The beam from the pump laser is directed onto the (100) or (111) sample facets, while the emission from the sample is registered in the orthogonal direction by a Ge:Ga detector inside the dipstick as well as by unstressed and stressed Ge:Ga detectors that are sensitive from 40 to $120 \mu \mathrm{m}$ and 120 to $210 \mu \mathrm{m}$, respectively. These detectors are mounted in a separate cryostat outside of the dipstick. The emission is analyzed with a FTIR spectrometer equipped with the Ge:Ga detectors. Combinations of infrared filters at liquid helium and at room temperature prevent pump radiation from reaching one of the detectors.

Photoionization pumping into the valence band is done with a transversely excited atmospheric $\mathrm{CO}_{2}$ laser that provides $1 \mathrm{MW}$ peak power in a $1 \mu \mathrm{s}$ pulse at a $5 \mathrm{~Hz}$ repetition rate. The laser is tunable in the range 114$135 \mathrm{meV}(9.2-10.9 \mu \mathrm{m})$. Intracenter pumping with photon energies in the range from 30 to $50 \mathrm{meV}$ is done with the FEL FELIX (formerly at the FOM Institute for Plasma Physics, Rijnhuizen, The Netherlands). Its pump pulses consist of 6- $\mu$ s-long macropulses at a repetition rate of $5 \mathrm{~Hz}$ and a peak power of up to $10 \mathrm{MW}$. Each macropulse in turn consists of $\sim 10$-ps-long micropulses with a spectral width of $\Delta \lambda / \lambda \approx 0.6 \%$ ( $\Delta \lambda$ is the FWHM) that are separated by $1 \mathrm{~ns}$. As has been shown for $n$-Si lasers, pumping with the frequency tunable FEL can be significantly more effective than photoionization pumping by a $\mathrm{CO}_{2}$ laser since it allows resonant pumping of excited states. This reduces the number of levels involved in the process of generating population inversion and consequently reduces recombination losses, for example, by nonradiative processes. In addition, the optical absorption on $1 \Gamma_{8}^{+} \rightarrow$ oddparity impurity transitions is more than an order of magnitude larger than that for photoionization, which enables more efficient pumping.

The relaxation time of the $1 \Gamma_{7}^{-}$and $1 \Gamma_{6}^{-}$states, which are, as we will see later, the most important states, are measured by a pump-probe technique. The $\mathrm{Si}: \mathrm{B}$ sample for these measurements is cut from the same material as one of the Si:B laser samples. It has a boron concentration $N_{B} \sim 2 \times$ $10^{15} \mathrm{~cm}^{-3}$ and a mean thickness of $400 \mu \mathrm{m}$. The Si:B surfaces, which face the pump and probe radiation, are polished and wedged in order to reduce interference effects in the sample. The experiments were carried out at the FEL of the Helmholtz Zentrum Dresden-Rossendorf. This FEL provides quasicontinuous radiation at a repetition rate of $13 \mathrm{MHz}$ and a pulse duration of approximately $10 \mathrm{ps}$. The experimental setup is described in detail elsewhere [24]. The high signal-to-noise ratio achievable with this setup allow us to significantly reduce the pump energy (down to $70 \mathrm{pJ}$ ) and accurately detect pump-induced transmission changes at the level of $0.3 \%$. Precise overlap of the FEL linewidth with the boron transitions $1 \Gamma_{8}^{+} \rightarrow 1 \Gamma_{7}^{-}, 1 \Gamma_{6}^{-}, 4 \Gamma_{8}^{-}$ insures optimal pumping of the probed states.

\section{RESULTS AND DISCUSSION}

In Fig. 2, a typical absorption spectrum of a Si:B sample is shown at a temperature of $5 \mathrm{~K}$. A number of absorption lines are clearly visible and these can all be assigned to transitions in Si:B, namely, the $1 \Gamma_{8}^{+} \rightarrow$ odd-parity impurity transitions (lines 1,2,3,4,4a,4b,5,6, .., 10; nomenclature according to Ref. [14]). The closely spaced 4, 4a, and $4 \mathrm{~b}$ lines are not resolved, because these lines are significantly concentration broadened in the Si:B sample. By tuning the frequency of the FEL, a similar absorption spectrum of the Si:B sample is obtained (Fig. 2), although with less spectral resolution because of the linewidth of the FEL. The slope in the spectrum is due to absorption by water vapor in the atmosphere and power variations of the FEL. Some of the absorption lines (marked with an asterisk) are caused by atmospheric water. Emission from the Si:B sample is observed only when pumping on the 4, 4a, 4b lines (Fig. 2), which correspond to the $1 \Gamma_{8}^{+} \rightarrow 1 \Gamma_{7}^{-}$, $1 \Gamma_{6}^{-}, 4 \Gamma_{8}^{-}$intracenter transitions (calculated oscillator strengths are $2.60 \times 10^{-2}, 3.76 \times 10^{-2}, 2.30 \times 10^{-3}$, correspondingly [16]). This is different from $n$-Si lasers where pumping from the ground state into several lowest odd-parity impurity transitions leads to laser emission [9]. The FWHM of the Si:B emission signal is approximately the same as that of the absorption line measured with the FEL, which indicates that each of the three pump lines leads to laser emission.

The Si:B laser emission spectrum (Fig. 3) consists of two lines at 1.740 and $1.748 \mathrm{THz}(172.3$ and $171.5 \mu \mathrm{m})$, which 


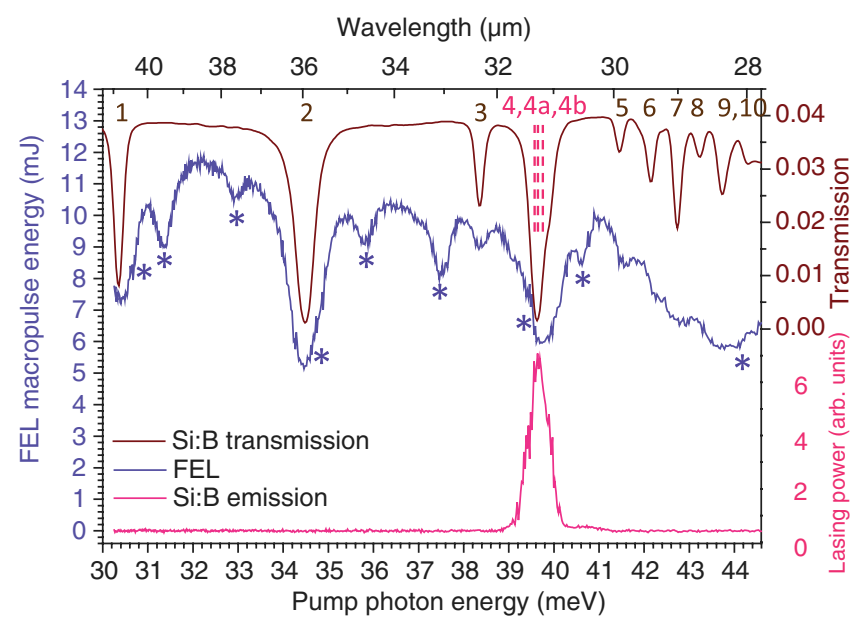

FIG. 2. Absorption and pump spectra of the Si:B laser samples. The uppermost curve is the transmission spectrum of a $400-\mu \mathrm{m}$ thick Si:B sample cut from the same crystal as the laser. It was measured at a temperature of $5 \mathrm{~K}$ with a FTIR spectrometer with $2 \mathrm{hPa}$ pressure. The impurity transitions are labeled $1,2,3, \ldots, 10$ according to Ref. [14]. The middle curve is an absorption spectrum of the $\mathrm{Si}: \mathrm{B}$ laser crystal at $5 \mathrm{~K}$ measured by tuning the wavelength of the FEL and detecting the transmitted radiation behind the Si:B sample. Note the correspondence between the absorption lines measured with the FTIR spectrometer and the FEL. They correspond to boron intracenter transitions originating from the boron ground state. The asterisks indicate positions of the strongest water vapor absorption lines. The lowest curve displays the $\mathrm{Si}: \mathrm{B}$ laser power as a function of the pump wavelength. Only pumping on the boron lines $4,4 a$, and $4 \mathrm{~b}$ results in laser emission.

are barely resolved due to the limited spectral resolution of the FTIR spectrometer $(\sim 5 \mathrm{GHz})$. The analysis of pump and emission spectra and comparison with the known $\mathrm{Si}: \mathrm{B}$ states allows identification of the involved boron states. The

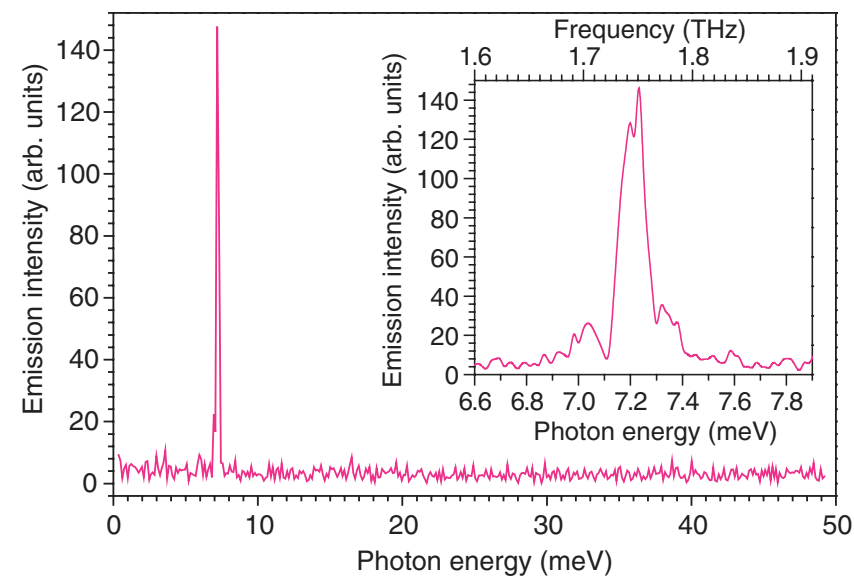

FIG. 3. Emission spectrum of the $\mathrm{Si}: \mathrm{B}$ laser when pumped at the boron line 4. The FEL pump photon flux is $4 \times 10^{25} \mathrm{~cm}^{-2} \mathrm{~s}^{-1}$. Two emission lines at 1.740 and $1.748 \mathrm{THz}$ are just resolved (inset). upper laser levels are the $1 \Gamma_{7}^{-}, 1 \Gamma_{6}^{-}$states, which are not resolved in the laser spectrum, and the $4 \Gamma_{8}^{-}$state. The lower laser level for all emission lines is the $2 \Gamma_{8}^{+}$state [Fig. 1]. The binding energy of the $2 \Gamma_{8}^{+}$state as derived from the laser spectrum is $-13.44 \mathrm{meV}$, which agrees very well with the experimental $(-13.44 \mathrm{meV}$ [25]) and calculated $(-13.41 \mathrm{meV}$ [25], $-13.34 \mathrm{meV}$ [15]) values. The laser scheme is an indirect indication that the $\Gamma_{7}^{+}$state is not the state with the largest binding energy, because in that case one would expect laser emission on the $1 \Gamma_{8}^{-} \rightarrow 1 \Gamma_{7}^{+}$ transition. In particular, absorption of pump radiation on the $1 \Gamma_{8}^{+} \rightarrow 1 \Gamma_{8}^{-}$(line 1 ) is as strong as on the $1 \Gamma_{8}^{+} \rightarrow 1 \Gamma_{7}^{-}$, $1 \Gamma_{6}^{-}, 4 \Gamma_{8}^{-}$(lines 4, 4a, 4b) (Fig. 2) and the relaxation time of the $1 \Gamma_{8}^{-}$state is longer than that of the $1 \Gamma_{7}^{-}, 1 \Gamma_{6}^{-}$states [23]. The coexistence of two emission lines and the large linewidth are indicators of low competition between the lasing transitions. This is caused by the low gain and small population of the upper laser level.

The lasing threshold is above $\sim 10^{24}$ photons $\mathrm{cm}^{-2} \mathrm{~s}^{-1}$ and depends on the pump photon energy (Fig. 4). Pumping directly at the center wavelength of the boron absorption line (4 and 4a: pump photon energy $39.6 \mathrm{meV}$ ) has a lower threshold than pumping in the wings of the absorption line (4b: $40.0 \mathrm{meV}$ ) (Fig. 4), because the absorption cross section for the pump radiation is smaller in the latter case. The relaxation time of the upper laser states is derived from pump-probe experiments by fitting the decay of the photoinduced transmission with an exponential function (Fig. 5). The finite duration of the pump and probe pulses is taken into account by convoluting the exponential decay with a Gaussian pulse of $\sim 10$ ps FWHM. The linewidth of the FEL is too large $(\Delta \lambda / \lambda \approx 0.6 \% \approx 2 \mu \mathrm{m})$ compared to the separation of these states $(\sim 0.2 \mu \mathrm{m})$ to allow measuring of the relaxation time of the $1 \Gamma_{7}^{-}, 1 \Gamma_{6}^{-}$, and $4 \Gamma_{8}^{-}$states individually. The relaxation time is $t=53 \pm 2 \mathrm{ps}$. It is independent of the pump energy across the whole range

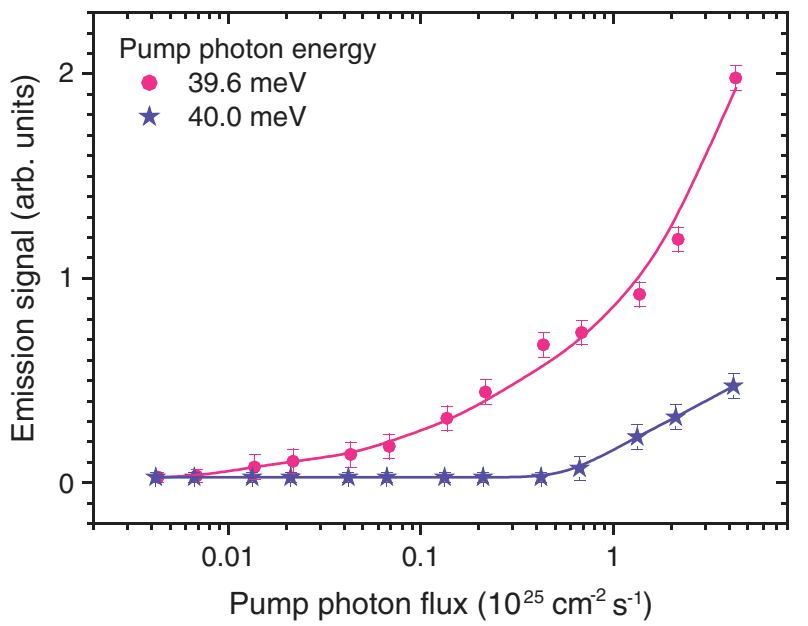

FIG. 4. Dependences of the emission from Si:B on the FEL pump photon flux for different photon pump energies, corresponding to boron absorption lines 4 and $4 \mathrm{~b}$. 


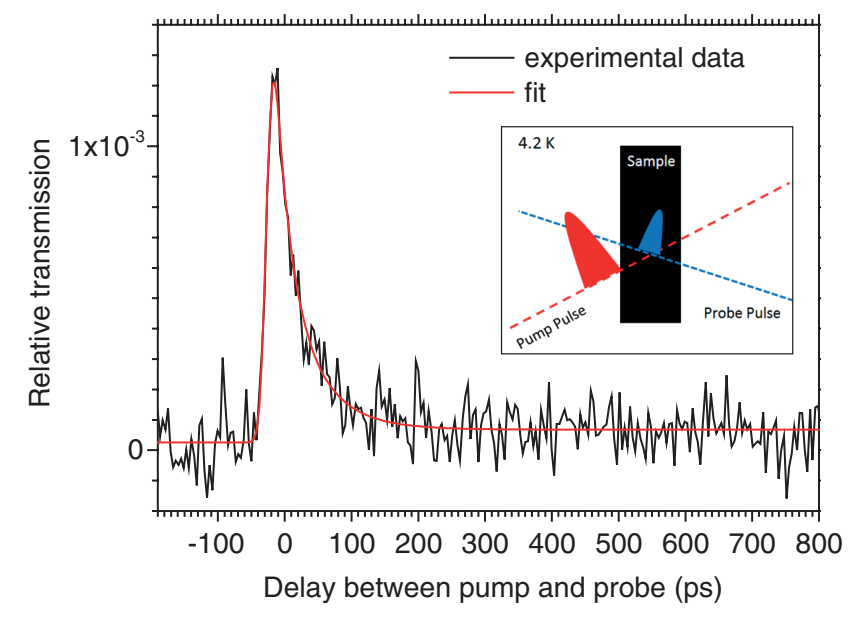

FIG. 5. Typical decay of the photoinduced transmission in Si:B. The pump and probe pulses have a Gaussian shape and a wavelength centred at the boron line $4(31.3 \mu \mathrm{m}$, resonant pumping in the upper laser state). The FEL pump photon energy is $113 \mathrm{pJ}$ per pulse. The inset is a sketch of the pump-probe experiment.

from 70 to $850 \mathrm{pJ}$ (Fig. 6), because at these low pump energies only relaxation from the excited into the ground state is important. Recombination processes become significant at pump energies above $2 \mathrm{~nJ}$ [24]. The relaxation time is shorter than the lifetimes of the odd-parity excited states in Si:B with larger binding energies [23] and it is significantly shorter than those of $n$-Si lasers, where the upper laser level has a typical lifetime between 100 and $250 \mathrm{ps}[26,27]$. The short lifetime of the upper laser level is also the reason for the rather high laser threshold. Only samples with a net boron concentration in the range $N_{B} \sim$ (1-3) $\times 10^{15} \mathrm{~cm}^{-3}$ exhibit laser emission while samples with $N_{B} \sim(4-5) \times 10^{15} \mathrm{~cm}^{-3}$ do not show emission. This is significantly lower than the maximum doping of $n$-Si lasers, which operate up to a concentration of $\sim 10^{16} \mathrm{~cm}^{-3}$ [9]. Because the spacing of the energy levels around the states involved in $\mathrm{Si}: \mathrm{B}$ lasing is smaller than in $n-\mathrm{Si}$,

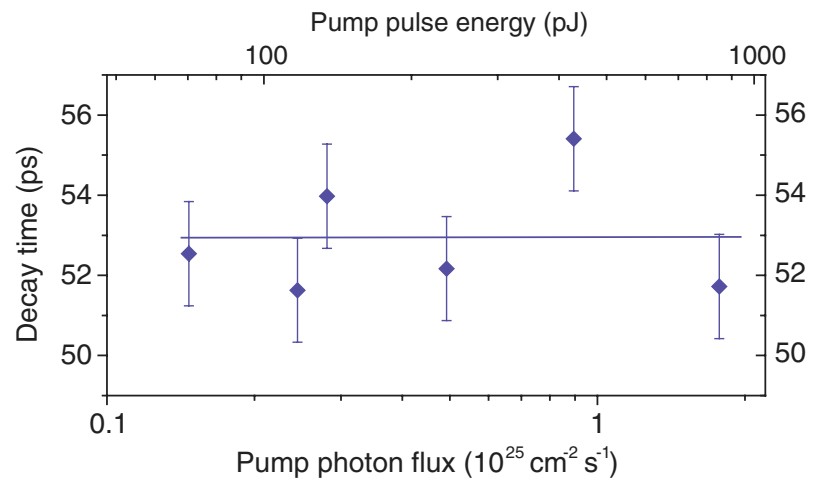

FIG. 6. Dependence of decay time of the Si:B sample (the same as in Fig. 5) on the pump pulse energy for pumping on line 4 ( $T \approx 5 \mathrm{~K})$. The straight line indicates the mean value. concentration broadening leads to overlap of the upper laser level with other states at lower concentration. This results in a shorter lifetime of the upper laser level and in less gain. The low gain is one of the reasons for the absence of stimulated emission when the samples are pumped with a $\mathrm{CO}_{2}$ laser and a photon flux density up to $\sim 2 \times$ $10^{25} \mathrm{~cm}^{-2} \mathrm{~s}^{-1}$ on the sample. The other reason is the lower pump efficiency of nonresonant pumping, because there are several relaxation paths for the excited carriers similar to those in $n$-Si [28]. Some of these do not involve the upper laser level, especially those utilizing even-parity excited states.

Also, no Raman-type stimulated emission is observed from any of investigated Si:B samples, even for maximum pump power from the FEL. This is different from $n$-Si lasers, where Raman lasing has been observed from all donors [9]. Assuming that the scattering strength of the resonant electronic Raman process in $\mathrm{Si}: \mathrm{B}$ is close to that of Si:P [17], one can expect stimulated Raman emission from the investigated $\mathrm{Si}: \mathrm{B}$ samples with a Stokes shift of $E_{S} \approx 23 \mathrm{meV}$, provided that the $1 \Gamma_{7}^{+}$ground state has a binding energy of approximately $22 \mathrm{meV}$. The fact that Raman lasing is not observed is another indication that the $1 \Gamma_{7}^{+}$state is very likely not located in the band gap of silicon. This is in contradiction to the Raman spectroscopy results discussed above [17,18]. Possibly, the observed spectral features in these spectra are a result of a resonance between the pump laser photon energy $(\lambda=1064 \mathrm{~nm}$, $E_{1064 \mathrm{~nm}} \approx 1.165 \mathrm{eV}$ ) and the sum of the indirect band gap of silicon $\left(E_{g} \approx 1.12 \mathrm{eV}\right)$ and the spin-orbit energy $E_{g}+\Delta_{\mathrm{SO}} \approx 1.164 \mathrm{eV}$. As has been shown by Wright and Mooradian, the Raman spectra of Si:P and Si:B have the same lines at 37.9 and $64.8 \mathrm{meV}$ [17]. While the line at $64.8 \mathrm{meV}$ is related to the zone-centered optical phonon $h \omega_{\text {LTO }}$, the line at $37.9 \mathrm{meV}$ might be related to the indirect band gap $E_{g}=E_{1064 \mathrm{~nm}}-37.9 \mathrm{meV}$. Then the lowerenergy line in the Si:B spectrum at $\sim 23.4 \mathrm{meV}$ evidently belongs to an electronic state that is not in the band gap $E_{g}$, but is resonant to the $1 \Gamma_{8}^{+}$continuum: $E_{B}=$ $E_{1064 \mathrm{~nm}}-23.4 \mathrm{meV} \approx 1.142 \mathrm{eV}>E_{g}$. An accurate interpretation of the spectra obtained by resonant Raman scattering requires experimental verification that would include both Stokes and anti-Stokes bands as well as a nonresonant excitation laser.

In order to investigate the issue of the $1 \Gamma_{7}^{+}$state, absorption spectra are measured with a FTIR spectrometer at temperatures up to $180 \mathrm{~K}$. Provided that the $1 \Gamma_{7}^{+}$state is in the band gap at $E_{1 \Gamma 7+} \approx-23 \mathrm{meV}$, it should be significantly populated at temperatures between 50 and $100 \mathrm{~K}$. This is similar to the absorption spectra of $n$-Si:As, whose $1 s(E)$ and $1 s\left(T_{2}\right)$ states are split off from the ground state by $\sim 21 \mathrm{meV}$ and where transitions from $1 s(E)$, $1 s\left(T_{2}\right)$ states into the higher-lying odd-parity states are observed at temperatures between 30 and $160 \mathrm{~K}$ with a maximum at around $75 \mathrm{~K}$. In $\mathrm{Si}: \mathrm{B}$, one would expect a 
series of absorption lines $1 \Gamma_{7}^{+} \rightarrow n \Gamma_{8}^{-}(n=1,2,4)$ and $1 \Gamma_{7}^{+} \rightarrow n \Gamma_{6}^{-}(n=2,3,4)$ from the thermally populated $1 \Gamma_{7}^{+}$state. However, we did not observe these transitions. Therefore, we conclude that the $1 \Gamma_{7}^{+}$state is very likely not located at the theoretically predicted binding energy in the band gap of silicon [15].

\section{CONCLUSION}

In conclusion, acceptors in silicon can be used to achieve terahertz lasing. Resonant optical excitation on the $1 \Gamma_{8}^{+} \rightarrow 1 \Gamma_{7}^{-}, 1 \Gamma_{6}^{-}, 4 \Gamma_{8}^{-}$intracenter transitions leads to laser emission on the $1 \Gamma_{6}^{-}, 1 \Gamma_{7}^{-}, 4 \Gamma_{8}^{-} \rightarrow 2 \Gamma_{8}^{+}$intracenter transitions at 1.740 and $1.748 \mathrm{THz}$, respectively. The lasing mechanism has some distinct differences compared to $n-\mathrm{Si}$ lasers. First, lasing occurs by optical pumping into the three boron states with the largest integral absorption cross section for the pump radiation. The lifetime of these states is rather short, approximately $50 \mathrm{ps}$. The $n$-Si lasers are most efficient when pumping into the states with the longest lifetime of about 100-200 ps. Also, Raman lasing was not observed from Si:B. The lasing mechanism has some implications for the energy level structure of $\mathrm{Si}: \mathrm{B}$, namely, that the spin-orbit split-off $1 \Gamma_{7}^{+}$ground state has a binding energy less than at least $-20 \mathrm{meV}$. This is confirmed by absorption spectroscopy of $\mathrm{Si}: \mathrm{B}$ at temperatures in the range $30-160 \mathrm{~K}$. The spectra do not show any absorption lines related to a $1 \Gamma_{7}^{+}$ground state that would be located in the energy gap of silicon. These results pave the way for $p$-type terahertz silicon lasers with other hydrogenlike acceptors such as $\mathrm{Al}, \mathrm{Ga}$ or In and novel investigations of carrier relaxation processes in $p$-type silicon.

\section{ACKNOWLEDGMENTS}

This work was supported by the German Federal Ministry of Research and Education (Grant No. 05K10KTD), the Netherlandse Organisatie voor Wetenschappelijk Onderzoek (NWO), and the Russian Foundation for Basic Research (Grants No. 14-02-00638 and No. 13-02-97116-povolzhie). N. D. acknowledges support by the Helmholtz Research School on Security Technologies. We thank R. Eichholz and U. Böttger for assistance with the experiments.

[1] W. Koechner and M. Bass, Solid-State Lasers (Springer, Berlin 2003).

[2] S. G. Pavlov, R. Kh. Zhukavin, E. E. Orlova, V. N. Shastin, A. V. Kirsanov, H.-W. Hübers, K. Auen, and H. Riemann, Stimulated Emission from Donor Transitions in Silicon, Phys. Rev. Lett. 84, 5220 (2000).

[3] S. G. Pavlov, H.-W. Hübers, J. N. Hovenier, T. O. Klaassen, D. A. Carder, P. J. Phillips, B. Redlich, H. Riemann, R. Kh. Zhukavin, and V. N. Shastin, Stimulated Terahertz Stokes
Emission of Silicon Crystals Doped with Antimony Donors, Phys. Rev. Lett. 96, 037404 (2006).

[4] T. J. Kippenberg, J. Kalkman, A. Polman, and K. J. Vahala, Demonstration of an Erbium-Doped Microdisk Laser on a Silicon Chip, Phys. Rev. A 74, 051802(R) (2006).

[5] L. G. Yang, C. H. Yeh, C. Y. Wong, C. W. Chow, F. G. Tseng, and H. K. Tsang, Stable and Wavelength-Tunable Silicon-Micro-Ring-Resonator-Based Erbium-Doped Fiber Laser, Opt. Express 21, 2869 (2013).

[6] O. Boyraz and B. Jalali, Demonstration of a Silicon Raman Laser Opt. Express 12, 5269 (2004).

[7] H. Rong, A. Liu, R. Jones, O. Cohen, D. Hak, R. Nicolaescu, A. Fang, and M. Paniccia, An All-Silicon Raman Laser, Nature (London) 433, 292 (2005).

[8] A. W. Fang, H. Park, O. Cohen, R. Jones, M. J. Paniccia, and J. E. Bowers, Electrically Pumped Hybrid AlGaInAsSilicon Evanescent Laser, Opt. Express 14, 9203 (2006).

[9] S. G. Pavlov, R. Kh. Zhukavin, V. N. Shastin, and H.-W. Hübers, The Physical Principles of Terahertz Silicon Lasers Based on Intracenter Transitions, Phys. Status Solidi B 250, 9 (2013).

[10] V. V. Tsyplenkov, E. V. Demidov, K. A. Kovalevsky, and V. N. Shastin, Relaxation of Excited Donor States in Silicon with Emission of Intervalley Phonons, Semiconductors 42, 1016 (2008).

[11] H.-W. Hübers, S. G. Pavlov and V. N. Shastin, Terahertz Lasers Based on Germanium and Silicon, Semicond. Sci. Technol. 20, S211 (2005).

[12] V. N. Shastin, R. Kh. Zhukavin, E. E. Orlova, S. G. Pavlov, M. H. Rümmeli, H.-W. Hübers, J. N. Hovenier, T. O. Klaassen, H. Riemann, I. V. Bradley, and A. F. G. van der Meer, Stimulated $\mathrm{THz}$ Emission from Group-V Donors in Silicon under Intracenter Photoexcitation Appl. Phys. Lett. 80, 3512 (2002).

[13] T. N. Adam, R. T. Troeger, S. K. Ray, P.-C. Lv, and J. Kolodzey, Terahertz Electroluminescence from BoronDoped Silicon Devices, Appl. Phys. Lett. 83, 1713 (2003).

[14] S. Zwerdling, K. J. Button, B. Lax, and L. M. Roth, Internal Impurity Levels in Semiconductors: Experiments in p-Type Silicon, Phys. Rev. Lett. 4173 (1960).

[15] R. Buczko and F. Bassani, Shallow Acceptor Resonant States in Si and Ge, Phys. Rev. B 45, 5838 (1992).

[16] B. Pajot, Optical Absorption of Impurities and Defects in Semiconducting Crystals: I. Hydrogen-like Centres (Springer, Berlin 2010).

[17] G. B. Wright and A. Mooradian, Raman Scattering from Donor and Acceptor Impurities in Silicon, Phys. Rev. Lett. 18, 608 (1967).

[18] J. M. Cherlow, R. L. Aggarwal, and B. Lax, Raman Scattering and Photoluminescence in Boron-Doped and Arsenic-Doped Silicon, Phys. Rev. B 7, 4547 (1973).

[19] H. R. Chandrasekhar, A. K. Ramdas, and S. Rodriguez, Stress-Induced Mixing of the Spin-Orbit-Split Acceptor States of Silicon, Phys. Rev. B 12, 5780 (1975).

[20] M. L. W. Thewalt, Even-Parity Acceptor Excited States in Si from Bound Exciton Two Hole Transitions, Solid State Commun. 23, 733 (1977).

[21] C. E. Jones, D. Schafer, W. Scott, and R. J. Hager, CarbonAcceptor Pair Centers (X Centers) in Silicon, J. Appl. Phys. 52, 5148 (1981). 
[22] P.-C. Lv, R. T. Troeger, T. N. Adam, S. Kim, J. Kolodzey, I. N. Yassievich, M. A. Odnoblyudov, and M. S. Kagan, Electroluminescence at 7 Terahertz from Phosphorus Donors in Silicon, Appl. Phys. Lett. 85, 22 (2004).

[23] N. Q. Vinh, B. Redlich, A. F. G. van der Meer, C. R. Pidgeon, P. T. Greenland, S. A. Lynch, G. Aeppli, and B. N. Murdin, Time-Resolved Dynamics of Shallow Acceptor Transitions in Silicon, Phys. Rev. X 3, 011019 (2013).

[24] N. Dessmann, S. G. Pavlov, V. N. Shastin, R. Kh. Zhukavin, V. V. Tsyplenkov, S. Winnerl, M. Mittendorff, N. V. Abrosimov, H. Riemann, and H.-W. Hübers, Time-Resolved Electronic Capture in n-Type Germanium Doped with Antimony, Phys. Rev. B 89, 035205 (2014).

[25] N. O. Lipari, A. Baldereschi, and M. L. W. Thewalt, Central Cell Effects on Acceptor Spectra in Si and Ge, Solid State Commun. 33, 277 (1980).
[26] N. Q. Vinh, P. T. Greenland, K. Litvinenko, B. Redlich, A. F. G. van der Meer, S. A. Lynch, M. Warner, A. M. Stoneham, G. Aeppli, D. J. Paul, C. R. Pidgeon, and B. N. Murdin, Silicon as a Model Ion Trap: Time Domain Measurements of Donor Rydberg States, Proc. Natl. Acad. Sci. U.S.A. 105, 10649 (2008).

[27] H.-W. Hübers, S. G. Pavlov, S. A. Lynch, T. Greenland, K. L. Litvinenko, B. Murdin, B. Redlich, A. F. G. van der Meer, H. Riemann, N. V. Abrosimov, P. Becker, H.-J. Pohl, R. Kh. Zhukavin, and V. N. Shastin, Isotope Effect on the Lifetime of the $2 p_{0}$ State in Phosphorus-Doped Silicon, Phys. Rev. B 88, 035201 (2013).

[28] S. G. Pavlov, H.-W. Hübers, P. M. Haas, J. N. Hovenier, T. O. Klaassen, R. Kh. Zhukavin, V. N. Shastin, D. A. Carder, and B. Redlich, Evidence of Noncascade Intracenter Electron Relaxation in Shallow Donor Centers in Silicon, Phys. Rev. B 78, 165201 (2008). 